LE ELEZIONI IN ITALIA

di Aldo Di VIRGILIO

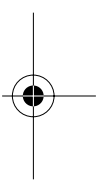




\section{LE 639 ELEZIONI "NON POLITICHE" DEL 2008: ONDA LUNGA PER IL CENTRODESTRA, OFFERTA DIFFORME, VOTO DIVISO}

Molto si è votato nel 2008. Oltre alle elezioni politiche generali si sono infatti tenute sei elezioni regionali (considerando tali anche le elezioni provinciali di Trento e Bolzano), 17 provinciali, 13 comunali in capoluoghi e 603 comunali in comuni non capoluogo. Si è trattato di oltre dieci milioni di elettori, la maggior parte dei quali alle prese con più schede (TAB. 1).

TAB. 1 - Elezioni comunali, provinciali e regionali 2008: numero di consultazioni ed elettori iscritti per aree geografiche.

\begin{tabular}{lcccccc}
\hline & \multicolumn{2}{c}{ Elezioni comunali } & \multicolumn{2}{c}{ Elezioni comunali } & \multicolumn{2}{c}{ Elezioni comunali } \\
\cline { 2 - 7 } & $\mathrm{n}$. & Elettori & $\mathrm{n}$. & Elettori & $\mathrm{n}$. & Elettori \\
\hline Nord & 167 & 1.250 .449 & 3 & 1.377 .322 & 4 & 1.992 .936 \\
Centro & 33 & 450.288 & 1 & 177.605 & & \\
Sud & 235 & 4.552 .544 & 5 & 4.765 .732 & 1 & 1.209 .079 \\
Isole & 181 & 1.746 .796 & 8 & 4.318 .555 & 1 & 4.572 .912 \\
& & & & & & \\
Italia & 604 & 8.000 .067 & 17 & 10.639 .214 & 6 & 7.774 .927 \\
\hline
\end{tabular}

Tali tornate di voto presentano tre principali motivi di interesse. Il primo è proprio la concomitanza fra elezioni diverse e dunque la possibilità degli elettori di esprimere scelte di voto difformi nelle diverse arene elettorali. Come si ricava dal calendario riportato nella TAB. 2, il grosso delle elezioni "non politiche" del 2008 si è svolto infatti contestualmente alle politiche del 13-14 aprile. Un secondo aspetto da considerare è l'offerta elettorale. La principale novità emersa in proposito nelle elezioni politiche - l'esordio delle liste PD e PDL e la costruzione di alleanze a 
perimetro ridotto - ha infatti avuto corso assai limitato su scala regionale e locale, dove i due nuovi partiti hanno riproposto le tradizionali alleanze "larghe". Nel voto regionale, provinciale e municipale, $\mathrm{PD}$ e $\mathrm{PDL}$ hanno cioè adottato scelte competitive fondate sulla costruzione di coalizioni ampie e tendenzialmente catch-all. Ciò ha reso partiti che si fronteggiavano come avversari nelle schede per la Camera e per il Senato (PDL e UDC a centrodestra, PD e Sinistra Arcobaleno a centrosinistra) in partner di coalizione nelle altre arene elettorali. Sul questo terreno l'UDC è stato il partito più mobile (come si addice alla sua ambizione di giocare, dal centro, la funzione di decisivo ago della bilancia). Il partito di Casini ha infatti oscillato tra la linea di opposizione di centro assunta per le elezioni politiche, il ritorno all'alleanza con i partiti di centrodestra (opzione, invero, largamente prevalente in sede locale e regionale) e la sperimentazione di nuove alleanze (com'è accaduto per le elezioni provinciale di Trento, dove l'UDC ha sostenuto la candidatura del presidente uscente di centrosinistra Lorenzo Dellai).

Il terzo elemento da considerare è l'evoluzione della composizione dei governi regionali e locali, ossia la continuità o il cambiamento del loro colore politico. La TAB. 3 consente di osservare in proposito l'entità del successo del centrodestra. Il centrodestra ha sottratto Friuli-Venezia Giulia e Abruzzo allo schieramento avversario, ha conquistato quattro province (Foggia più le tre province siciliane governate fino al voto del giugno 2008 dal centrosinistra), ha perso il controllo dei comuni di Sondrio e Vicenza, ma assunto il controllo dell'amministrazione di Brescia, Messina e, soprattutto, la guida del comune Roma con Alemanno.

Le elezioni regionali: novità normative, nuovi governatori di centrodestra in Sicilia, Friuli e Abruzzo, conferma al ribasso dei governi autonomisti

Sistemi elettorali. In due delle regioni chiamate al voto (Friuli-Venezia Giulia e Valle d'Aosta) si è votato con regole elettorali in parte diverse da quelle in vigore per le elezioni precedenti. In Friuli ciò è avvenuto per la terza volta consecutiva. Dopo le elezioni del 1993 - le ultime con la proporzionale utilizzata dal 1964 - la legge è stata infatti modificata una prima volta alla vigilia delle elezioni del 1998 (introduzione di una soglia del 4,5\% dei voti per l'accesso al collegio unico regionale e all'assegnazione dei seggi-resti), poi alla vigilia delle elezioni del 2003 (applicazione della normativa transitoria prevista per le elezioni a statuto ordinario a seguito della legge costituzionale $2 / 2001$ e introduzione, dunque, dell'elezione diretta del presidente della giunta regionale) e infine alla vigilia delle elezioni del 2008 (legge regionale 226/2007). Le novità principali sono state l'eliminazione del listino regionale e l'introduzione di uno sbarramento del $4 \%$ per le liste in corsa da sole o per le liste appartenenti a coalizioni che non abbiano raggiungano il 15\% dei voti. Per le liste appartenenti a coalizioni con oltre il $15 \%$ dei voti validi, la soglia è stata fissata all' $1,5 \%$ dei voti validi. 


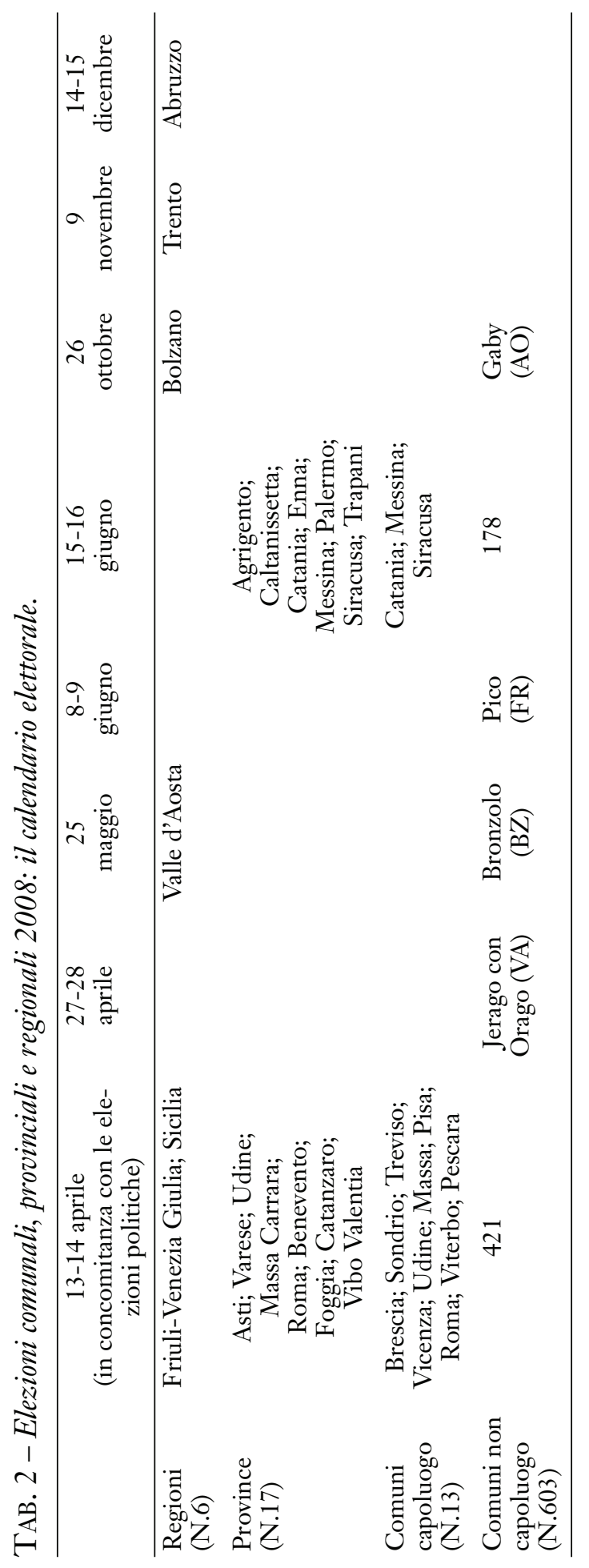

170 

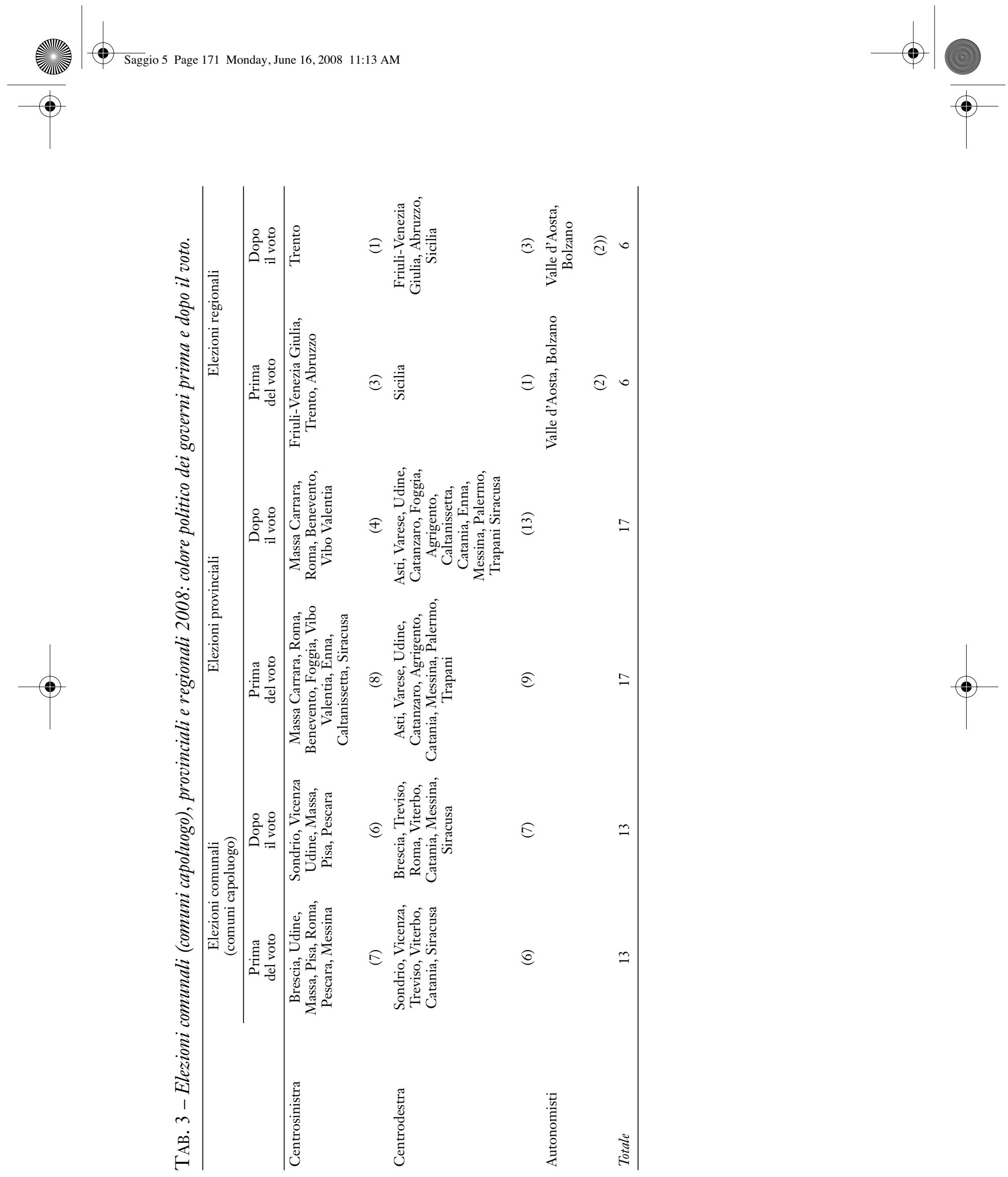
La nuova legge ha fissato inoltre all' $1 \%$ la soglia per la minoranza slovena nelle circoscrizioni di Trieste, Gorizia e Udine e stabilito che siano donne il 40\% dei candidati di ogni lista, così come tre assessori dei dieci che, secondo Statuto, dovranno comporre la giunta regionale.

Per quanto riguarda la Valle d'Aosta, la 1.r. 22/2007 ha introdotto alcune novità nella normativa per l'elezione del Consiglio (che rimane di impianto proporzionale con formula Hare del quoziente naturale e dei più alti resti). La legge prevede la possibilità che due o più liste sottoscrivano un programma elettorale comune e ha introdotto un premio di maggioranza tale da assicurare il 60\% dei seggi (ossia 21 seggi) alla lista o alla coalizione con più del $50 \%$ dei voti. Nel caso in cui nessuna lista o coalizione abbia ottenuto almeno 18 seggi è previsto un ballottaggio fra le due liste o coalizioni più votate. A quella vincente viene assegnato il 51\% dei seggi (18 seggi). L'ingresso in consiglio è subordinato alla conquista di almeno due quozienti pieni (e non più uno come fra il 1993 e il 2007). L'elettore può esprimere fino a tre voti di preferenza all'interno della lista prescelta.

Prima del voto sono state modificate anche le norme elettorali della provincia autonoma di Trento, ma unicamente con riferimento a ineleggibilità, incompatibilità, composizione della giunta. Sono rimaste invece invariate le norme elettorali in senso stretto definite nel 2002-03: elezione diretta del Presidente; elezione del consiglio su base proporzionale corretta da un premio di maggioranza eventuale (in nessun caso superiore a 24 seggi su 35); possibilità di esprimere fino a tre preferenze di lista; impossibilità di disgiungere voto per il Presidente e voto di lista.

Per completezza si ricorda che la Regione Sicilia, a seguito della legge costituzionale $2 / 2001$, aveva allineato il proprio sistema elettorale alla normativa vigente nelle regioni a statuto ordinario dopo le riforme del 1995 e del 1999. La nuova legge - utilizzata per la prima volta nel 2001 con l'elezione diretta di Cuffaro contro Orlando - era stata poi modificata dalla legge regionale $7 / 2005$, la quale confermò l'elezione diretta del Presidente, dimezzò il numero dei candidati dei listini maggioritari e introdusse una soglia di sbarramento del $5 \%$ dei voti su scala regionale per le liste dei candidati all'Assemblea Regionale. La nuova legge fu applicata per la prima volta alle regionali 2006 (elezione di Cuffaro contro la Borsellino; sensibile riduzione della frammentazione partitica nell'ARS). Invariata anche la normativa elettorale per la provincia autonoma di Bolzano (dichiarazione di appartenenza a un gruppo linguistico da parte dei consiglieri, composizione della giunta in rapporto alla consistenza dei gruppi linguistici presenti in Consiglio, elezione del consiglio su base proporzionale, possibilità di esprimere fino a quattro preferenze, elezione del Presidente da parte del Consiglio) e per la regione Abruzzo.

Sicilia, Friuli, Abruzzo: i nuovi governatori di centrodestra. In Sicilia e in Friuli le elezioni regionali si sono svolte in concomitanza con le politiche del 13-14 aprile. In Sicilia e in Abruzzo si è trattato di elezioni anticipate, in entrambi i casi per cause di carattere politico-giudiziario e per sopraggiunte dimissioni del presidente di giunta (Cuffaro, UDC, in Sicilia; Del Turco, PD, in Abruzzo). In tutte e tre le regioni il centrodestra ha vinto la contesa, eleggendo, rispettivamente, Lombardo (MPA), Tondo (PDL) e Chiodi (PDL) alla testa dei nuovi governi regionali. 
In Sicilia, come in Friuli, la concomitanza fra regionali e politiche ha contribuito a una crescita della partecipazione di 7-8 punti percentuali rispetto alle regionali precedenti (ТАВB. 4 e 5). Fra regionali e politiche il numero dei votanti differisce: un piccolo numero di elettori ha infatti evitato la scheda regionale; alle politiche il numero di votanti risulta pertanto più elevato. Si spiega invece con la normativa relativa agli elettori residenti all'estero lo scarto tra numero di elettori registrati negli elenchi per le regionali e numero di elettori registrati negli elenchi per le politiche. La differenza fra i due elettorati è costituita da quegli elettori che per le elezioni politiche risultano iscritti nelle liste degli italiani residenti all'estero (in vista della controversa elezione dei 18 parlamentari delle quattro ripartizioni estere) e che per le elezioni regionali sono invece iscritti in loco, risultando così, in larga parte, elettori astensionisti.

Come si è detto, gli elettori siciliani e friulani si sono trovati a scegliere fra alleanze partitiche diverse da quelle definite per le elezioni politiche. In Sicilia questa diversa offerta è stata l'esito di un negoziato nazionale. Gli autonomisti dell'MPA, guidati dall'ex-UDC Raffaele Lombardo, hanno infatti subordinato la propria adesione al centrodestra nazionale a formato ridotto (PDL-Lega-MPA) a due condizioni relative allo scacchiere siciliano: la candidatura alla presidenza della giunta regionale - posizione cui sembrava essere favorito l'esponente di Forza Italia Gianfranco Micciché - per Lombardo; l'estensione della coalizione regionale all'UDC (partito avversario della coalizione PDL-Lega-MPA alla Camera e della coalizione PDL-MPA al Senato in Sicilia). Analoga variabilità verticale delle alleanze si è avuta nello schieramento del centrosinistra, con la ricomposizione della frattura fra PD e Sinistra Arcobaleno a sostegno della candidatura di Anna Finocchiaro.

Il verdetto delle urne è stato netto: tanto il candidato quanto la coalizione di centrodestra hanno ottenuto più del doppio dei voti andati ottenuti dalla candidata $\mathrm{e}$ alla coalizione di centrosinistra (ТАB. 6). Al di fuori delle due coalizioni non è rimasto che un quasi $5 \%$ dei voti, distribuito fra le due candidature di estrema destra (DestraFiamma tricolore e Forza nuova) e la candidatura "anti-politica" degli "Amici di Beppe Grillo", lista certificata dall'attore satirico genovese e dal suo blog.

Lombardo ha ottenuto la maggioranza assoluta dei voti in tutte e nove le province dell'isola, con variazioni comprese fra il $70 \%$ ottenuto nel feudo di Catania e il 54\% di Enna (provincia che, assieme a Ragusa, costituisce tradizionalmente la zona di maggior forza della sinistra siciliana) (ТАВ. 7).

La contestualità fra voto regionale e voto politico consente di osservare in quale misura la diversa posta in palio istituzionale, le diverse regole del gioco e la costruzione di una diversa offerta partitica e di coalizione abbiano influenzato il comportamento degli elettori siciliani. La TAB. 8 pone a confronto valori assoluti e percentuali di voto per le liste di partito nelle due elezioni del 13-14 aprile. I saldi sono assai rilevanti. I due partiti maggiori, che nella scheda per la Camera catalizzano i consensi elettorali anche grazie all'appello al "voto utile", vedono fortemente ridotto il proprio seguito elettorale nella scheda regionale. Per il PDL, in particolare, si tratta di una differenza di quasi 500mila voti, pari a circa un terzo dei voti ricevuti alla Camera. 


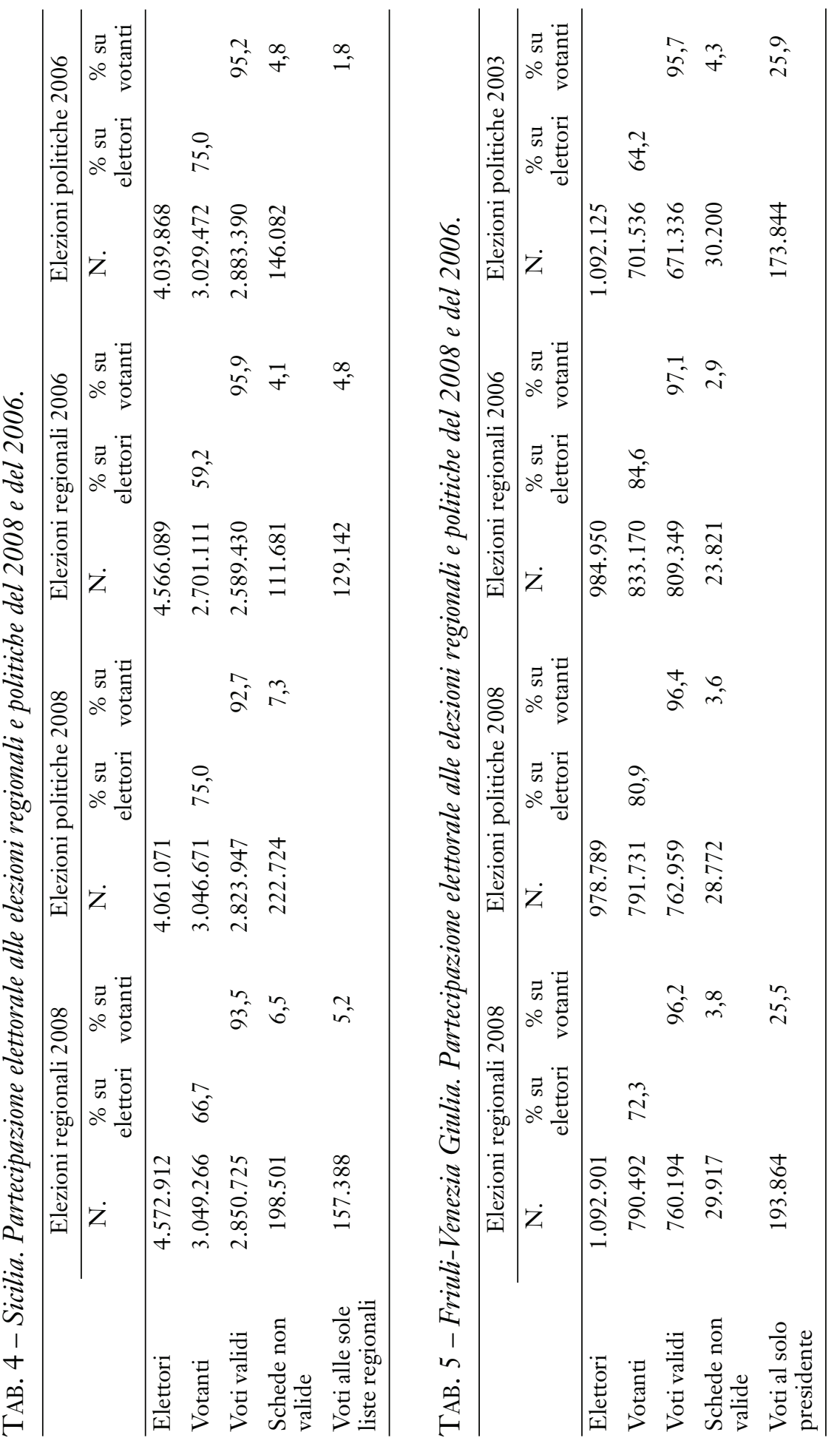


TAB. 6 - Sicilia. Riepilogo dei risultati delle elezioni regionali del 13-14 aprile 2008.

\begin{tabular}{|c|c|c|c|c|c|c|c|}
\hline $\begin{array}{l}\text { Candidati } \\
\text { presidenti }\end{array}$ & Voti & $\%$ & $\begin{array}{c}\text { Seggi } \\
\text { maggioritari }\end{array}$ & Liste & Voti & $\%$ & $\begin{array}{l}\text { Seggi } \\
\text { proporz. }\end{array}$ \\
\hline \multirow[t]{6}{*}{ Raffaele Lombardo } & 1.862 .959 & 65,4 & 1 & & & & \\
\hline & & & & PDL & 900.149 & 33,4 & 34 \\
\hline & & & & UDC & 336.826 & 12,5 & 11 \\
\hline & & & & $\begin{array}{l}\text { MPA-Alleati per il } \\
\text { Sud }\end{array}$ & 375.587 & 13,9 & 15 \\
\hline & & & & $\begin{array}{l}\text { Lombardo } \\
\text { presidente Sicilia } \\
\text { libera e forte }\end{array}$ & 119.892 & 4,4 & \\
\hline & & & & $\begin{array}{l}\text { Democratici } \\
\text { autonomisti }\end{array}$ & 101.449 & 3,8 & \\
\hline Centrodestra & & & & & 1.833 .903 & 68,1 & 60 \\
\hline \multirow[t]{5}{*}{ Anna Finocchiaro } & 866.044 & 30,4 & 1 & & & & \\
\hline & & & 8 & $\mathrm{PD}$ & 505.420 & 18,8 & 20 \\
\hline & & & & Sinistra Arcobaleno & 131.213 & 4,8 & \\
\hline & & & & $\begin{array}{l}\text { Anna Finocchiaro } \\
\text { Presidente per la } \\
\text { Sicilia }\end{array}$ & 83.700 & 3,1 & \\
\hline & & & & IDV & 49.726 & 1,8 & \\
\hline Centrosinistra & & & & & 770.059 & 28,6 & \\
\hline \multirow[t]{2}{*}{ Sonia Alfano } & 69.551 & 2,4 & & & & & \\
\hline & & & & $\begin{array}{l}\text { Amici di Beppe } \\
\text { Grillo - con Sonia } \\
\text { Alfano Presidente }\end{array}$ & 46.396 & 1,7 & \\
\hline \multirow{2}{*}{$\begin{array}{l}\text { Ruggero Benedetto } \\
\text { Italo Razza }\end{array}$} & 45.605 & 1,6 & & & & & \\
\hline & & & & $\begin{array}{l}\text { La Destra - } \\
\text { Fiamma tricolore }\end{array}$ & 39.143 & 1,4 & \\
\hline \multirow{2}{*}{$\begin{array}{l}\text { Giuseppe Bonanno } \\
\text { Conti }\end{array}$} & 6.606 & & & & & & \\
\hline & & & & Forza nuova & 3.876 & 0,1 & \\
\hline Totale generale & 2.850 .725 & 100 & 10 & & 2.693 .377 & 100 & 80 \\
\hline
\end{tabular}


TAB. 7 - Sicilia. Elezioni regionali 13-14 aprile 2008. Voto per Lombardo e Finocchiaro per provincia (valori percentuali).

\begin{tabular}{lcccc}
\hline & Lombardo & Finocchiaro & Altri $\left(^{*}\right)$ & Totale \\
\hline Agrigento & & & & \\
Caltanissetta & 63,8 & 33,7 & 2,5 & 100 \\
Catania & 61,6 & 33,4 & 5,0 & 100 \\
Enna & 70,2 & 24,2 & 5,6 & 100 \\
Messina & 53,7 & 40,5 & 5,8 & 100 \\
Palermo & 66,6 & 28,6 & 4,8 & 100 \\
Ragusa & 65,6 & 30,6 & 3,8 & 100 \\
Siracusa & 60,1 & 36,2 & 3,7 & 100 \\
Trapani & 62,1 & 34,3 & 3,6 & 100 \\
Totale & 64,8 & 32,0 & 3,2 & 100 \\
\hline
\end{tabular}

(*) Si tratta della somma dei voti conseguiti da Alfano, Razza e Conti.

Il voto per il PDL ha così rappresentato i quasi tre quarti dei consensi di centrodestra alla Camera e soltanto la metà nel voto regionale. Questo andamento risente soprattutto del successo del voto a favore di Lombardo e degli autonomisti dell'MPA. Sia per l'"effetto trascinamento" connesso all'elezione diretta a una carica monocratica di governo, sia per il forte radicamento territoriale dell'MPA in alcune zone dell'isola, il saldo positivo tra voti regionali e voti per la Camera è assai consistente (si tratta di oltre $150 \mathrm{mila}$ voti in più se si considera la sola lista MPA e di 280mila voti in più se si tiene conto anche della lista "Lombardo presidente - Sicilia libera e forte"). In termini percentuali si tratta di uno scarto di oltre 10 punti (rispetto ai voti validi: si tenga conto in proposito del minor numero di voti validi di lista espressi alle regionali rispetto alle politiche). Per l'UDC la differenza fra le due arene elettorali è più contenuta, con un saldo positivo di tre punti, pari a circa 70 mila voti.

Nello schieramento di centrosinistra le grandezze variano in modo diverso. Pur esprimendo la candidatura a governatore - e malgrado anche la Finocchiaro, com'è ormai consuetudine, abbia presentato una lista personale a sostegno della propria candidatura - il PD ha registrato alle elezioni regionale un saldo negativo di oltre 200mila voti, pari a quasi 7 punti percentuali (quattro se si tiene conto dei voti della Lista Finocchiaro). Il saldo è negativo anche per l'IDV (il che potrebbe approssimare il vero peso del partito di Di Pietro, una volta deflazionato il risultato per la Camera dal surplus di consensi connesso all'apparentamento concesso da Veltroni). La Sinistra Arcobaleno, invece, ottiene alle regionali quasi il doppio dei voti ricevuti nel voto per la Camera. 
TAB. 8 - Sicilia, elezioni 13-14 aprile 2008. Il voto ai partiti per le elezioni regionali e per le elezioni politiche.

\begin{tabular}{|c|c|c|c|c|}
\hline \multirow{2}{*}{ Liste } & \multicolumn{2}{|c|}{ Regionali 2008} & \multicolumn{2}{|c|}{ Politiche 2008} \\
\hline & Voti & $\%$ & Voti & $\%$ \\
\hline PDL & 900.149 & 33,4 & 1.316 .868 & 46,6 \\
\hline UDC & 336.826 & 12,5 & 264.953 & 9,4 \\
\hline MPA-Alleati per il Sud & 375.587 & 13,9 & 217.169 & 7,7 \\
\hline $\begin{array}{l}\text { Lombardo Presidente Sicilia libera e } \\
\text { forte }\end{array}$ & 119.892 & 4,4 & & \\
\hline Democratici autonomisti & 101.449 & 3,8 & & \\
\hline Coalizione Lombardo & 1.833 .903 & 68,1 & 1.798 .990 & 63,7 \\
\hline PD & 505.420 & 18,8 & 718.494 & 25,5 \\
\hline Sinistra Arcobaleno & 131.213 & 4,8 & 74.457 & 2,6 \\
\hline $\begin{array}{l}\text { Anna Finocchiaro Presidente per la } \\
\text { Sicilia }\end{array}$ & 83.700 & 3,1 & & \\
\hline IDV & 49.726 & 1,8 & 95.773 & 3,4 \\
\hline Coalizione Finocchiaro & 770.059 & 28,6 & 888.724 & 31,4 \\
\hline $\begin{array}{l}\text { Amici di Beppe Grillo - con Sonia } \\
\text { Alfano Presidente }\end{array}$ & 46.396 & 1,7 & & \\
\hline La Destra - Fiamma tricolore & 39.143 & 1,4 & 57.256 & 2,0 \\
\hline PS & & & 17.365 & 0,6 \\
\hline Sinistra critica & & & 11.252 & 0,4 \\
\hline $\begin{array}{l}\text { Ass. difesa della vita - Aborto? No, } \\
\text { grazie }\end{array}$ & & & 11.169 & 0,4 \\
\hline PCL & & & 10.893 & 0,4 \\
\hline PLI & & & 8.727 & 0,3 \\
\hline Forza nuova & 3.876 & 0,1 & 7.778 & 0,3 \\
\hline $\begin{array}{l}\text { Unione democratica per i } \\
\text { consumatori }\end{array}$ & & & 7.714 & 0,3 \\
\hline Per il Bene comune & & & 4.079 & 0,1 \\
\hline Totale & 2.693 .377 & 100 & 2.823 .947 & 100 \\
\hline
\end{tabular}

Nell'altra regione in cui le elezioni regionali si sono svolte lo stesso giorno delle elezioni politiche - il Friuli-Venezia Giulia - le differenze sono state assai più contenute. In questo caso, com'era già avvenuto nel 2003, l'aspetto più rilevante è l'elevata propensione degli elettori friuliani a votare per il solo candidato presidente, senza indicazione di un voto di lista, né, di conseguenza, di voti di preferenza per il consiglio. Questa opzione di voto è adottata da ben un elettore su 
quattro (si torni alla TAB. 5 e si veda la TAB. 9). Ciò significa che, in valore assoluto, entrambi i candidati non hanno difficoltà a far meglio delle proprie coalizioni. Il punto di maggiore interesse è però il seguente: a differenza di quanto era accaduto nelle elezioni del 2003 il consenso percentuale dei candidati presidenti è pressoché identico al consenso percentuale delle rispettive coalizioni partitiche. Ciò significa che Illy e Tondo sono beneficiari in egual misura del voto al solo presidente espresso dagli elettori. Nel 2003, invece, la candidatura di Riccardo Illy era stato il fattore decisivo della competizione (tre punti percentuali in più rispetto alla propria coalizione, contro i due decimi in meno del $2008 ; 7,5 \%$ dei voti validi per la lista personale del candidato contro il 5\% del 13-14 aprile 2008).

TAB. 9 - Friuli-Venezia Giulia. Riepilogo dei risultati delle elezioni regionali del 13-14 aprile 2008.

\begin{tabular}{|c|c|c|c|c|c|c|c|}
\hline $\begin{array}{l}\text { Candidati } \\
\text { presidenti }\end{array}$ & Voti & $\%$ & $\begin{array}{c}\text { Seggi } \\
\text { maggioritari }\end{array}$ & Liste & Voti & $\%$ & $\begin{array}{c}\text { Seggi } \\
\text { proporz. }\end{array}$ \\
\hline \multirow[t]{5}{*}{ Renzo Tondo } & 409.430 & 53,8 & 1 & & & & \\
\hline & & & 1 & PDL & 187.075 & 33,1 & 21 \\
\hline & & & & Lega Nord & 73.239 & 12,9 & 8 \\
\hline & & & & UDC & 34.840 & 6,2 & 4 \\
\hline & & & & $\begin{array}{l}\text { Partito } \\
\text { pensionati }\end{array}$ & 8.561 & 1,5 & 1 \\
\hline Centrodestra & & & & & 303.715 & 53,6 & 34 \\
\hline \multirow[t]{6}{*}{ Riccardo Illy } & 351.064 & 46,2 & 1 & & & & \\
\hline & & & & $\mathrm{PD}$ & 169.597 & 29,9 & 15 \\
\hline & & & & $\begin{array}{l}\text { Sinistra } \\
\text { Arcobaleno }\end{array}$ & 32.401 & 5,6 & 3 \\
\hline & & & & $\begin{array}{l}\text { Cittadini per il } \\
\text { Presidente }\end{array}$ & 28.855 & 5,1 & 2 \\
\hline & & & & IDV & 25.414 & 4,5 & 2 \\
\hline & & & & $\begin{array}{l}\text { Slovenska } \\
\text { Skupnost }\end{array}$ & 7.008 & 1,2 & 1 \\
\hline Centrosinistra & & & & & 262.915 & 46,4 & 23 \\
\hline Totale generale & 760.494 & 100 & 3 & & 566.630 & 100 & 57 \\
\hline
\end{tabular}

In Friuli, come si è detto, le differenze fra voto regionale e voto politico sono molto più contenute rispetto alla Sicilia (TAB. 10). I segni dei saldi sono però gli stessi: flessione per i partiti maggiori, lieve crescita per i partiti minori (soprattutto per la Sinistra Arcobaleno che ottiene per le regionali quasi il 50\% in più dei voti conquistati per la Camera). A livello di coalizione si sono registrati invece andamenti diversi (e diversi anche rispetto a quanto osservato in Sicilia). In termini percentuali il centrodestra ha ottenuto nelle due arene pressoché lo stesso 
risultato, mentre il centrosinistra alle regionali ha ricevuto circa sette punti e mezzo in più alle regionali, probabilmente per il combinato disposto della minor dispersione (alle politiche liste quali PCL, Sinistra critica, socialisti e Bene comune avevano totalizzato quasi il $2 \%$ dei voti validi) e della maggiore ampiezza della coalizione.

ТАВ. 10 - Friuli-Venezia Giulia, elezioni 13-14 aprile 2008. Il voto ai partiti per le elezioni regionali e per le elezioni politiche.

\begin{tabular}{|c|c|c|c|c|}
\hline \multirow{2}{*}{ Liste } & \multicolumn{2}{|c|}{ Regionali 2008} & \multicolumn{2}{|c|}{ Politiche 2008} \\
\hline & Voti & $\%$ & Voti & $\%$ \\
\hline PDL & 187.075 & 33,1 & 264.988 & 34,7 \\
\hline Lega Nord & 73.239 & 12,9 & 99.496 & 13,0 \\
\hline UDC & 34.840 & 6,2 & 46.051 & 6,0 \\
\hline Partito pensionati & 8.561 & 1,5 & & \\
\hline Coalizione Tondo & 303.715 & 53,7 & 410.535 & 53,7 \\
\hline PD & 169.597 & 29,9 & 239.346 & 31,4 \\
\hline Sinistra Arcobaleno & 32.401 & 5,6 & 23.278 & 3,1 \\
\hline Cittadini per il Presidente & 28.855 & 5,1 & & \\
\hline IDV & 25.414 & 4,5 & 32.746 & 4,3 \\
\hline Slovenska Skupnost & 7.008 & 1,2 & & \\
\hline Coalizione Illy & 263.275 & 46,3 & 295.370 & 38,7 \\
\hline La Destra & & & 22.585 & 3,0 \\
\hline La Lista dei Grilli parlanti & & & 8.403 & 1,1 \\
\hline PS & & & 4.271 & 0,6 \\
\hline PCL & & & 3.882 & 0,5 \\
\hline Sinistra critica & & & 3.609 & 0,5 \\
\hline Per il Bene comune & & & 3.394 & 0,4 \\
\hline Ass. difesa della vita - Aborto? No, grazie & & & 3.049 & 0,4 \\
\hline Forza nuova & & & 2.925 & 0,4 \\
\hline Unione democratica per i consumatori & & & 2.328 & 0,3 \\
\hline PLI & & & 2.607 & 0,3 \\
\hline Totale & 566.630 & 100 & 762.958 & 100 \\
\hline
\end{tabular}


Il centrodestra, come si è detto, si è affermato anche in Abruzzo. Le elezioni regionali anticipate si sono svolte in questo caso a metà dicembre. Ciò ha fatto uscire la regione, com'era già accaduto per il Molise, dal calendario elettorale delle regioni a statuto ordinario. Ne è risultata una consistente flessione della partecipazione elettorale: ha votato infatti appena il 53\% degli aventi diritto, quasi 16 punti in meno rispetto al 2005 (ТАB. 11).

Il centrodestra, che la stessa offerta delle politiche (PDL e MPA, senza UDC) con l'aggiunta della lista personale del candidato Chiodi ("Rialzati Abruzzo"), ha ottenuto un risultato non lontano dal 50\% dei voti validi (TAB 12). Il candidato presidente ha ottenuto oltre otto punti in più rispetto al candidato del centrodestra nel 2005. In picchiata, invece, il consenso per il centrosinistra: dal $58 \%$ del 2005 al $43 \%$ del 2008 nel voto al candidato presidente; dal 54\% al 45\% nel voto alle liste di candidati per il consiglio. È così nonostante il centrosinistra si presentasse con il formato della coalizione amplia (PD, IDV e partiti dell'ex Sinistra Arcobaleno). Le componenti della Sinistra Arcobaleno hanno ritrovato libertà d'azione e anche di ricombinazione (Verdi e Sinistra Democratica, ad esempio, si sono presentati assieme come La Sinistra). Per il centrosinistra il risultato più significativo rispetto alle elezioni politiche di otto mesi prima è stato però la caduta del voto al PD (dal 33,5\% di aprile al 19,6\% di dicembre) e la crescita dell'IDV (dal 7\% al 15\%). Una redistribuzione dei pesi elettorali certo connessa alle vicende locali che hanno determinato il ricorso alle elezioni anticipate e che hanno inciso sul livello della partecipazione elettorale. Ma anche una misura della capacità di Di Pietro di incalzare l'alleato maggiore e di sollecitarne l'elettorato, dopo aver tratto vantaggio dalle dinamiche proprie della mini-coalizione PD-IDV.

Valle d'Aosta, Bolzano, Trento: tra (risicate) conferme e fatti nuovi. Il voto regionale nelle periferie linguistiche ha confermato i governi autonomisti uscenti. Dalle urne sono però usciti spostamenti di voto anche consistenti. In Valle d'Aosta (TABB. 13 e 14) l'Union Valdôtaine ha ottenuto 17 seggi su 35 alla testa della Coalizione autonomista, che ha raggiunto 23 seggi grazie alle liste centriste Stella Alpina-UDC e Federazione Autonomista (formazioni che nel 2003, con un'unica lista, avevano ottenuto un seggio in più). Sono cresciute di un seggio rispetto al 2003 sia la Coalizione autonomista progressista guidata dal PD (l'Arcobaleno valdostano, con il 5,6\% dei voti validi, è però rimasto escluso dal consiglio), sia il PDL (quattro seggi contro i tre di Forza Italia nel 2003).

A Bolzano la SVP, pur mantenendo la maggioranza assoluta dei seggi, ha perso 7 punti rispetto al 2003 e per la prima volta in elezioni provinciali è scesa al di sotto del $50 \%$ dei voti validi. La SVP si è trovata incalzata da Die Freiheitlichen, lista che ha triplicato i voti del 2003 e ha eletto cinque consiglieri, tre in più (ТАBB 15 e 16). Il PDL ha perso in voti e seggi rispetto al risultato di AN e Forza Italia nel 2003. Entra invece nel consiglio provinciale di Bolzano, con un eletto, la Lega Nord Südtirol. 


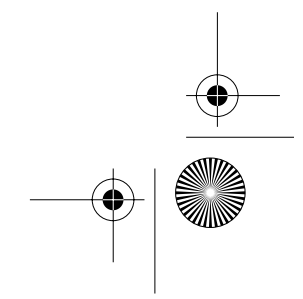


ТАВ. 12 - Abruzzo. Riepilogo dei risultati delle elezioni regionali del 14-15 dicembre 2008.

\begin{tabular}{|c|c|c|c|c|c|c|c|}
\hline $\begin{array}{l}\text { Candidati } \\
\text { presidenti }\end{array}$ & Voti & $\%$ & $\begin{array}{c}\text { Seggi } \\
\text { maggioritari }\end{array}$ & Liste & Voti & $\%$ & $\begin{array}{c}\text { Seggi } \\
\text { proporz. }\end{array}$ \\
\hline \multirow{5}{*}{$\begin{array}{l}\text { Gianni Chiodi } \\
P D L\end{array}$} & 295.371 & 48,8 & 8 & & & & \\
\hline & & & & PDL & 190.919 & 35,2 & 15 \\
\hline & & & & Rialzati Abruzzo & 40.256 & 7,4 & 3 \\
\hline & & & & MPA & 18.040 & 3,3 & 1 \\
\hline & & & & Liberalsocialisti & 7.753 & 1,4 & - \\
\hline Centrodestra & & & & & 256.968 & 47,4 & 19 \\
\hline \multirow{8}{*}{$\begin{array}{l}\text { Carlo Costantini } \\
\text { Costantini presidente }\end{array}$} & 258.199 & 42,7 & 1 & & & & \\
\hline & & & & $\mathrm{PD}$ & 106.410 & 19,6 & 7 \\
\hline & & & & IDV & 81.557 & 15,0 & 5 \\
\hline & & & & $\mathrm{RC}$ & 15.435 & 2,9 & 1 \\
\hline & & & & La Sinistra & 12.054 & 2,2 & 1 \\
\hline & & & & PDCI & 9.955 & 1,9 & 1 \\
\hline & & & & PS & 9.387 & 1,7 & - \\
\hline & & & & $\begin{array}{l}\text { Democratici per } \\
\text { l'Abruzzo }\end{array}$ & 7.507 & 1,4 & - \\
\hline Centrosinistra & & & & & 242.305 & 44,7 & 15 \\
\hline \multirow{2}{*}{$\begin{array}{l}\text { Rodolofo } \\
\text { De Laurentis } \\
U D C \text {-UDEUR }\end{array}$} & 32.604 & 5,4 & & & & & \\
\hline & & & & UDC-UDEUR & 30.452 & 5,6 & 2 \\
\hline \multirow{2}{*}{$\begin{array}{l}\text { Teodoro Buon- } \\
\text { tempo } \\
\text { La Destra }\end{array}$} & 11.514 & 1,9 & & & & & \\
\hline & & & & La Destra & 9.597 & 1,8 & \\
\hline \multirow{2}{*}{$\begin{array}{l}\text { Ilaria Del Biondo } \\
\text { Partito Comunista } \\
\text { dei Lavoratori }\end{array}$} & 4.625 & 0,8 & & & & & \\
\hline & & & & PCL & 2.018 & 0,4 & \\
\hline \multirow{2}{*}{$\begin{array}{l}\text { Angelo } \\
\text { Di Prospero } \\
\text { Per il Bene comune }\end{array}$} & 2.791 & 0,5 & & & & & \\
\hline & & & & Bene comune & 1.237 & 0,2 & \\
\hline Totale generale & 605.104 & 100 & 9 & & 542.577 & 100 & 36 \\
\hline
\end{tabular}




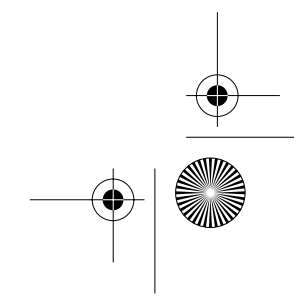


TAB. 14 - Valle d'Aosta. Riepilogo dei risultati delle elezioni regionali del 25 maggio 2008.

\begin{tabular}{lccc}
\hline \multicolumn{1}{c}{ Liste } & Voti & $\%$ & Seggi \\
\hline Union Valdôtaine & 32.614 & 44,4 & 17 \\
Stella Alpina-UDC & 8.370 & 11,4 & 4 \\
Féderation autonomiste & 4.536 & 6,2 & 2 \\
Coalizione autonomista & 44.520 & 62,0 & 23 \\
& & & \\
VdA Vive -Renouveau Valdôtaine & 9.169 & 12,5 & 5 \\
PD & 6.840 & 9,3 & 3 \\
Arcobaleno VdA & 4.121 & 5,6 & - \\
Coalizione autonomista progressista & 20.131 & 27,4 & 8 \\
& & & \\
PDL & 7.826 & 10,7 & 4 \\
Centrodestra & 7.826 & 10,7 & 4 \\
Totale & 73.476 & 100 & 35 \\
\hline
\end{tabular}

La Lega Nord ha ottenuto un risultato assai consistente anche a Trento. Ha sorpassato infatti il PDL ed è passata da due a sei seggi in consiglio. Il centrosinistra guidato da Dellai ha ottenuto un largo successo (TABB. 17 e 18). Rispetto al 2003 ha registrato però un calo di oltre 3 punti percentuali, anche se Dellai si è afferma con oltre 20 punti di scarto sul candidato del centrodestra Divina (nel 2003 lo scarto sul forzista Andreotti era stato di oltre 30 punti). Dellai ha anche ottenuto un ottimo risultato con la propria lista personale (Unione per il Trentino - Dellai Presidente) che sfiora il 18\% (mentre il PD si è fermato al 21,6\%). Un aspetto dell'offerta e del risultato che attenua la rilevanza nazionale del voto di Trento, di cui molto si è parlato per il sostegno a Dellai accordato, con l'avallo di Casini, dall'UDC trentina. 


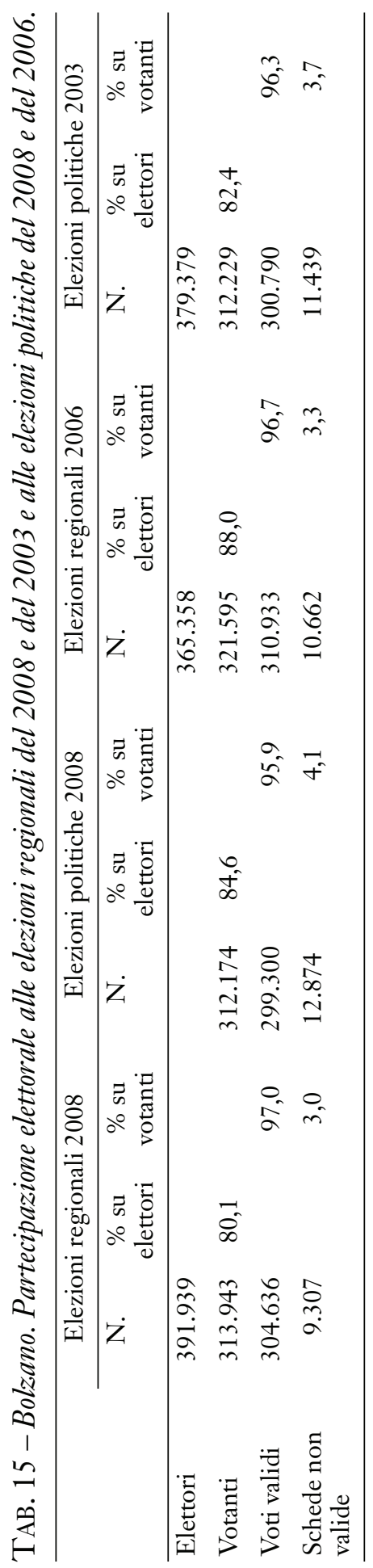

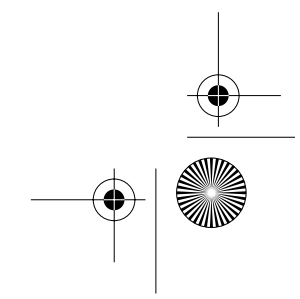


TAB. 16 - Bolzano. Riepilogo dei risultati delle elezioni regionali del 26 ottobre 2008.

\begin{tabular}{lcccc}
\hline Liste & Voti & $\%$ & Seggi & $\%$ \\
\hline Südtiroler Volkspartei & 146.555 & 48,1 & 18 & 51,4 \\
Die Freiheitlichen & 43.615 & 14,3 & 5 & 14,3 \\
PDL & 25.297 & 8,3 & 3 & 8,6 \\
PD & 18.141 & 6,0 & 2 & 5,7 \\
Verdi & 17.745 & 5,8 & 2 & 5,7 \\
Süd-Tiroler Freiheit & 14.888 & 4,9 & 2 & 5,7 \\
Union Für Südtirol & 7.048 & 2,3 & 1 & 2,9 \\
Lega Nord Südtirol & 6.413 & 2,1 & 1 & 2,9 \\
Unitalia Movimento Iniziativa Sociale & 5.689 & 1,9 & 1 & 2,9 \\
IDV & 5.009 & 1,6 & - & \\
UDC & 3.792 & 1,2 & - & \\
Bürgerbewegung & 3.622 & 1,2 & - & \\
Ladins Dolomites & 3.334 & 1,1 & - & \\
Sinistra Alto Adige / Linke Für Südtirol & 2.226 & 0,7 & - & \\
PDCI & 1.262 & 0,4 & - & \\
Totale & 304.636 & 100 & 35 & \\
\hline
\end{tabular}




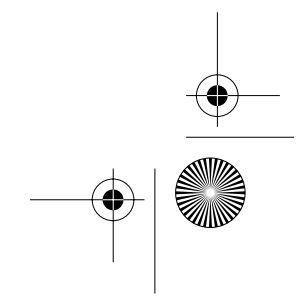


TАВ. 18 - Trento. Riepilogo dei risultati delle elezioni regionali del 9 novembre 2008.

\begin{tabular}{|c|c|c|c|c|c|c|c|}
\hline $\begin{array}{l}\text { Candidati } \\
\text { presidenti }\end{array}$ & Voti & $\%$ & $\begin{array}{c}\text { Seggi } \\
\text { maggioritari }\end{array}$ & Liste & Voti & $\%$ & $\begin{array}{c}\text { Seggi } \\
\text { proporz. }\end{array}$ \\
\hline \multirow{8}{*}{ Lorenzo Dellai } & 165.046 & 57,0 & 1 & & & & \\
\hline & & & & PD & 59.219 & 21,6 & 8 \\
\hline & & & & $\begin{array}{l}\text { Unione per il Trentino - } \\
\text { Dellai Presidente }\end{array}$ & 49.035 & 17,9 & 6 \\
\hline & & & & PATT & 23.336 & 8,5 & 3 \\
\hline & & & & Verdi & 7.579 & 2,8 & 1 \\
\hline & & & & IDV & 7.474 & 2,7 & 1 \\
\hline & & & & Leali per il Trentino & 6.449 & 2,4 & - \\
\hline & & & & Union Autonomista Ladina & 3.205 & 1,2 & - \\
\hline Centrosinistra & & & & & 156.297 & 57,0 & 20 \\
\hline \multirow[t]{11}{*}{ Sergio Divina } & 105.696 & 36,5 & 1 & & & & \\
\hline & & & & Lega Nord & 38.536 & 14,1 & 6 \\
\hline & & & & PDL & 33.597 & 12,3 & 5 \\
\hline & & & & $\begin{array}{l}\text { Lista Civica per Divina } \\
\text { Presidente }\end{array}$ & 11.832 & 4,3 & 1 \\
\hline & & & & Autonomisti Valli Unite & 5.846 & 2,4 & - \\
\hline & & & & Pensionati & 3.597 & 1,3 & - \\
\hline & & & & Fassa & 1.669 & 0,6 & - \\
\hline & & & & Fiamma Tricolore & 1.652 & 0,6 & - \\
\hline & & & & La Destra & 1.643 & 0,6 & - \\
\hline & & & & Inquilini Case Popolari & 1.375 & 0,5 & - \\
\hline & & & & Autonomisti popolari & 803 & 0,3 & - \\
\hline Centrodestra & & & & & 100.550 & 36,7 & 12 \\
\hline \multirow[t]{3}{*}{ Nerio Giovanazzi } & 8.401 & 2,9 & 1 & & & & \\
\hline & & & & Amministrare il Trentino & 4.429 & 1,6 & - \\
\hline & & & & Giovani per il Trentino & 2.717 & 0,9 & - \\
\hline $\begin{array}{l}\text { Totale coalizione } \\
\text { Giovanazzi }\end{array}$ & & & & & 7.146 & 2,5 & - \\
\hline \multirow[t]{2}{*}{ Remo Andreolli } & 5.653 & 1,9 & & & & & \\
\hline & & & & Democratici per il Trentino & 5.363 & & \\
\hline \multirow[t]{2}{*}{ Agostino Catalano } & 3.354 & 1,2 & & & & & \\
\hline & & & & La Sinistra per il Trentino & 3.190 & & \\
\hline \multirow[t]{2}{*}{ Gianfranco Valduga } & 1.448 & 0,5 & & & & & \\
\hline & & & & PDCI & 1.373 & & \\
\hline Totale generale & 289.598 & 100 & 3 & & 273.919 & 100 & 32 \\
\hline
\end{tabular}

Le elezioni provinciali: il centrodestra riconquista la Sicilia; il PD, in difficoltà, mantiene Roma

Le elezioni provinciali 2008 hanno interessato, in due tornate diverse, 17 unità amministrative: tutte le province siciliane eccetto Ragusa, più un gruppo di nove province tra cui Roma. La TAB. 19, in cui sono riportati i dati relativi alla partecipazione elettorale, mostra lo scarto fra il primo e il secondo gruppo, scarto che registra non solo i tradizionali differenziali di partecipazione nelle diverse latitudini della penisola, ma anche le conseguenze dell'effetto calendario (si torni alla 
TAB. 2). Si segnala, in particolare, la partecipazione inferiore al $50 \%$ degli aventi diritto a Palermo e Caltanissetta e compresa fra il 50\% e il 60\% a Agrigento, Trapani e Enna.

Le TABв. 20 e 21 riportano i risultati per l'elezione dei presidenti. In 12 dei 17 casi la contesa si è risolta al primo turno. Si tratta delle otto province siciliane più Vibo Valentia, Benevento, Udine e Varese. In 10 di questi 12 casi è uscito eletto il candidato del centrodestra. Si tratta in tutti i casi di un centrodestra "largo", comprendente i centristi dell'UDC. Nessuno degli eletti al primo turno è un uscente. L'unico presidente riconfermato è stato Angeli (PD) a Massa Carrara, il quale ha centrato l'obiettivo al secondo turno. Al turno di ballottaggio il centrosinistra ha avuto la meglio anche Roma con Zingaretti, che al secondo turno subisce il ritorno del candidato del centrodestra Antoniozzi (indietro di 10 punti al primo turno e di appena tre al secondo).

TАВ. 19 - Elezioni provinciali 2008: partecipazione elettorale (17 casi).

\begin{tabular}{|c|c|c|c|c|c|c|c|c|c|}
\hline \multirow{4}{*}{ Provincia } & \multirow{4}{*}{$\frac{\text { Elettori }}{\mathrm{n} .}$} & \multirow{4}{*}{$\frac{\text { Votanti }}{\%}$} & \multicolumn{5}{|c|}{ Primo turno } & \multirow{3}{*}{\multicolumn{2}{|c|}{$\begin{array}{c}\text { Secondo turno } \\
\text { Voti validi } \\
\text { Ballottaggio }\end{array}$}} \\
\hline & & & \multirow{2}{*}{\multicolumn{2}{|c|}{$\begin{array}{l}\text { Voti validi } \\
\text { Presidente }\end{array}$}} & \multirow{2}{*}{\multicolumn{2}{|c|}{$\begin{array}{c}\text { Voti validi } \\
\text { Liste }\end{array}$}} & \multirow{3}{*}{$\begin{array}{c}\text { Voti al solo } \\
\text { Presidente } \\
\% \\
\text { (sugli } \\
\text { elettori) }\end{array}$} & & \\
\hline & & & & & & & & & \\
\hline & & & $\mathrm{n}$. & $\begin{array}{c}\% \\
\text { (sugli } \\
\text { elettori) }\end{array}$ & $\mathrm{n}$. & $\begin{array}{c}\% \\
\text { (sugli } \\
\text { elettori) }\end{array}$ & & $\mathrm{n}$. & $\%$ \\
\hline Asti & & 76,6 & 129.908 & & 106.484 & & & 84.518 & \\
\hline Varese & & 79,7 & 542.063 & & 503.818 & & & & \\
\hline Udine & 487.934 & & 329.870 & & 299.150 & & 30.720 & & \\
\hline $\begin{array}{l}\text { Massa } \\
\text { Carrara }\end{array}$ & & 76,1 & 125.884 & & 114.782 & & & 87.217 & \\
\hline Roma & & 74,7 & 2.397 .414 & & 2.275 .441 & & & 1.945 .331 & \\
\hline Benevento & & 72,6 & 187.933 & & 183.554 & & & & \\
\hline Foggia & & 69,4 & 374.225 & & 361.084 & & & 245.156 & \\
\hline Catanzaro & & 66,4 & 211.727 & & 202.344 & & & 134.386 & \\
\hline $\begin{array}{l}\text { Vibo } \\
\text { Valentia }\end{array}$ & & 62,7 & 98.459 & & 96.570 & & & & \\
\hline Agrigento & 472.988 & 52,0 & 226.451 & 47,9 & 221.099 & 46,7 & 5.352 & & \\
\hline Caltanissetta & 276.638 & 49,8 & 130.183 & 47,1 & 127.126 & 46,0 & 3.057 & & \\
\hline Catania & 953.022 & 63,9 & 530.191 & 55,6 & 494.512 & 51,9 & 35.679 & & \\
\hline Enna & 193.784 & 57,2 & 102.627 & 53,0 & 99.401 & 51,3 & 3.226 & & \\
\hline Messina & 590.247 & 68,4 & 364.768 & 61,8 & 352.233 & 59,7 & 12.535 & & \\
\hline Palermo & 1.093 .670 & 41,1 & 413.553 & 37,8 & 402.524 & 36,8 & 11.029 & & \\
\hline Siracusa & 356.174 & 63,1 & 204.106 & 57,3 & 194.266 & 54,5 & 9.840 & & \\
\hline Trapani & 382.032 & 56,9 & 198.989 & 52,1 & 192.932 & 50,5 & 6.057 & & \\
\hline
\end{tabular}


ТАВ 20 - Elezioni provinciali 2008. Presidenti di provincia eletti al primo turno (12 casi).

\begin{tabular}{|c|c|c|c|}
\hline Provincia & $\begin{array}{l}\text { Presidente } \\
\text { eletto }\end{array}$ & $\%$ & Sostegno elettorale \\
\hline Varese & Galli & 64,1 & PDL; Lega Nord; UDC \\
\hline Udine & Fontanini & 55,4 & PDL; Lega Nord; UDC \\
\hline Benevento & Cimitile & 55,1 & $\begin{array}{l}\text { PD; UDEur; Progetto Sannio; Costituente di centro; IDV; Sannio } \\
\text { Democratico; PS; Sinistra Arcobaleno }\end{array}$ \\
\hline Vibo Valentia & De Nisi & 58,5 & $\begin{array}{l}\text { Con e per la gente; PD; Partecipazione democratica; Democratici vibonesi; } \\
\text { Riformisti; Popolari Democratici; I Democratici di } 360 \text { gradi; Centro è } \\
\text { libertà; Solidarietà democratica; Democratici vibonesi per De Nisi; IDV }\end{array}$ \\
\hline Agrigento & D’Orsi & 67,9 & $\begin{array}{l}\text { Democratici autonomisti; PDL; MPA; UDC; Sicilia forte e libera-Lombardo } \\
\text { presidente }\end{array}$ \\
\hline Caltanissetta & Federico & 63,5 & $\begin{array}{l}\text { DC-PLI; PDL; La Destra-Alleanza siciliana; MPA; UDC; Sicilia forte e } \\
\text { libera-Lombardo presidente }\end{array}$ \\
\hline Catania & Castiglione & 77,6 & $\begin{array}{l}\text { Democratici Autonomisti; PDL; Lista per Castiglione presidente; MPA; Nello } \\
\text { Musumeci per la provincia; Sicilia forte e libera-Lombardo presidente; UDC }\end{array}$ \\
\hline Enna & Monaco & 53,9 & $\begin{array}{l}\text { UDC; PDL; MPA; Fiamma tricolore; La Destra-Alleanza siciliana; Monaco } \\
\text { presidente; Alternativa democratica }\end{array}$ \\
\hline Messina & Ricevuto & 75,4 & $\begin{array}{l}\text { Alleanza Tirrenica; Democratici Autonomisti; Destra Sicilia per la Libertà; } \\
\text { Gioventù della Liberta'; MPA; il Centro con D'Alia; PDL; MPA; PRI; } \\
\text { Provincia punto freccia; Ricevuto presidente-Forza Azzurri; UDC }\end{array}$ \\
\hline Palermo & Avanti & 72,3 & $\begin{array}{l}\text { PDL; UDC; MPA; La Destra; Alleanza per la libertà-PRI; L’Aquilone-PLI- } \\
\text { Movimento per la libertà }\end{array}$ \\
\hline Siracusa & Bono & 68,6 & PDL; MPA; UDC; Alleanza Azzurra Sicilia \\
\hline Trapani & Turano & 65,8 & PDL; MPA, UDC, Ettore Fieramosca -Fratelli d'Italia \\
\hline
\end{tabular}

ТАВ 21 - Elezioni provinciali 2008. Presidenti di provincia eletti al ballottaggio (5 casi).

\begin{tabular}{|c|c|c|c|c|c|c|c|c|}
\hline Provincia & $\begin{array}{l}\text { Presidente } \\
\text { eletto }\end{array}$ & $\begin{array}{c}\% \\
\left(I I^{\circ} \text { t. }\right)\end{array}$ & $\begin{array}{c}\% \\
\left(I^{\circ} \text { t. }\right)\end{array}$ & Sostegno elettorale & $\begin{array}{l}\text { Candidato } \\
\text { sconfitto }\end{array}$ & $\begin{array}{c}\% \\
\left(I{ }^{\circ} \text { t. }\right)\end{array}$ & $\begin{array}{c}\% \\
\left(I^{\circ} \text { t. }\right)\end{array}$ & $\begin{array}{l}\text { Sostegno } \\
\text { elettorale }\end{array}$ \\
\hline Asti & Armosino & 58,0 & 42,0 & PDL; Lega Nord & Peretti & 42,0 & 26,4 & PD; IDV \\
\hline $\begin{array}{l}\text { Massa } \\
\text { Carrara }\end{array}$ & Angeli & 55,4 & 41,5 & $\begin{array}{l}\text { PD; PS-Socialisti-Laici- } \\
\text { Repubblicani; IDV }\end{array}$ & Bondi & 44,6 & 32,3 & PDL \\
\hline Roma & Zingaretti & 51,5 & 46,9 & $\begin{array}{l}\text { PD; Sinistra Arcobaleno; } \\
\text { IDV, Lista Zingaretti; Lista } \\
\text { Bonino Radicali; Unione } \\
\text { Democratica per i } \\
\text { Consumatori; Forza Roma; } \\
\text { Avanti Lazio }\end{array}$ & Antoniozzi & 48,5 & 37,1 & $\begin{array}{l}\text { PDL; Lista civica } \\
\text { Antoniozzi; PRI; } \\
\text { Lista del Grillo } \\
\text { parlante; MPA }\end{array}$ \\
\hline Foggia & Pepe & 54,0 & 37,4 & $\begin{array}{l}\text { PDL; Alleanza per la } \\
\text { Capitanata; La Capitanata } \\
\text { prima di tutto; Lista del } \\
\text { presidente Pepe; Oltre il } \\
\text { Polo; Azione sociale } \\
\text { Mussolini; UDC; Rosa } \\
\text { Bianca; Destra FT; } \\
\text { Santianell O8 presidente; I } \\
\text { Socialisti; Partito pensionati }\end{array}$ & Campo & 46,0 & 42,8 & $\begin{array}{l}\text { PD; PS; Sinistra } \\
\text { Arcobaleno; IDV; } \\
\text { Mov. diritti } \\
\text { Capitanata }\end{array}$ \\
\hline Catanzaro & Ferro & 60,0 & 45,9 & $\begin{array}{l}\text { PDL; MPA; NPSI; } \\
\text { Popolari europei per la } \\
\text { Libertà; Per la Calabria; la } \\
\text { Destra-Fiamma Tricolore; } \\
\text { PRI; Democrazia e } \\
\text { centralità; }\end{array}$ & Amato & 40,0 & 35,7 & $\begin{array}{l}\text { PD; Popolari } \\
\text { Democratici; IDV; } \\
\text { PS; Provincia } \\
\text { d'amare; Verdi; } \\
\text { Grandinetti per il } \\
\text { Lametino }\end{array}$ \\
\hline
\end{tabular}

Nota: i nomi in corsivo si riferiscono a presidenti uscenti; le liste in corsivo si riferiscono a collegamenti fra liste e candidati sopraggiunti al secondo turno. 
Elezioni comunali nei capoluoghi: la sfida di Roma, i molti ballottaggi, il turn-over dei sindaci

Le elezioni comunali 2008 hanno visto il rinnovo della carica di sindaco in 13 capoluoghi di provincia. Anche in questo caso la partecipazione (TAB. 22) ha risentito oltre che delle specificità territoriali anche del calendario elettorale. Dei sindaci uscenti soltanto tre hanno voluto o potuto ripresentarsi agli elettori. Due Gobbo a Treviso e D'Alfonso a Pescara - sono stati confermati al primo turno (TAB. 23). Il terzo - il democratico Neri a Massa - è invece giunto in testa dopo il primo turno, ma è poi risultato sconfitto in un ballottaggio che ha visto un consistente calo di partecipazione (si torni alla TAB. 22) e il successo del candidato della sinistra radicale Pucci (TAB. 24). Il centrodestra si è aggiudicato quasi tutti i successi di primo turno (nel caso di Catania e Siracusa con ampio margine). La sfida di Roma ha catalizzato l'attenzione al turno di ballottaggio. Rutelli, che al primo turno aveva sopravanzato Alemanno di cinque punti ottenendo il 45,8\% dei voti, è stato sconfitto 54 a 46 nel ballottaggio. Per il centrosinistra (e per il PD in particolare) si è trattato di una sconfitta più bruciante di quella subita due settimane prima alle politiche, in quanto sopraggiunta piuttosto inaspettata.

TAB. 22 - Elezioni comunali nei capoluoghi 2008: partecipazione elettorale (13 casi).

\begin{tabular}{|c|c|c|c|c|c|c|c|c|c|}
\hline \multirow{3}{*}{ Comune } & \multirow{3}{*}{$\frac{\text { Elettori }}{\mathrm{n} .}$} & \multirow{3}{*}{$\begin{array}{c}\text { Votanti } \\
\%\end{array}$} & \multicolumn{5}{|c|}{ Primo turno } & \multirow{2}{*}{\multicolumn{2}{|c|}{$\begin{array}{c}\text { Secondo t urno } \\
\text { Voti validi } \\
\text { Ballottaggio }\end{array}$}} \\
\hline & & & \multicolumn{2}{|c|}{$\begin{array}{l}\text { Voti validi } \\
\text { Sindaco }\end{array}$} & \multicolumn{2}{|c|}{$\begin{array}{l}\text { Voti validi } \\
\text { Liste }\end{array}$} & \multirow{2}{*}{$\begin{array}{c}\begin{array}{c}\text { Voti al } \\
\text { solo } \\
\text { Sindaco }\end{array} \\
\% \\
\text { (sugli } \\
\text { elettori) }\end{array}$} & & \\
\hline & & & n. & $\begin{array}{c}\% \\
\text { (sugli } \\
\text { elettori) }\end{array}$ & n. & $\begin{array}{c}\% \\
\text { (sugli } \\
\text { elettori) }\end{array}$ & & n. & $\begin{array}{c}\% \\
\text { (sugli } \\
\text { elettori) }\end{array}$ \\
\hline Brescia & 145.429 & 84,7 & 118.971 & 81,8 & 102.645 & 68,7 & 11,2 & & \\
\hline Sondrio & 18.740 & 79,8 & 14.512 & 77,4 & 12.864 & 66,5 & 8,8 & 12.507 & 66,7 \\
\hline Treviso & 66.279 & 79,4 & 51.186 & 77,2 & 45.699 & 66,8 & 8,3 & & \\
\hline Vicenza & 86.993 & 81,1 & 68.717 & 79,0 & 62.351 & 70,2 & 7,3 & 54.770 & 63,0 \\
\hline \multicolumn{10}{|l|}{ Udine } \\
\hline Massa & 59.438 & 82,5 & 47.236 & 79,5 & 44.823 & 74,8 & 4,1 & 34.523 & 58,1 \\
\hline Pisa & 74.369 & 79,8 & 57.203 & 76,9 & 52.370 & 68,9 & 6,5 & 41.152 & 55,3 \\
\hline Roma & 2.350 .875 & 73,6 & 1.663 .154 & 70,7 & 1.533 .992 & 63,2 & 5,4 & 1.459 .781 & 62,1 \\
\hline Viterbo & 52.078 & 85,7 & 43.389 & 83,3 & 41.106 & 78,2 & 4,4 & 35.446 & 68,1 \\
\hline Pescara & 105.484 & 80,4 & 81.964 & 77,7 & 74.377 & 68,8 & 7,2 & & \\
\hline Catania & 269.297 & 68,2 & 166.274 & 61,7 & 155.756 & 57,8 & 3,9 & & \\
\hline Messina & 204.023 & 75,6 & 68.386 & 70,8 & 64.981 & 67,9 & 2,9 & & \\
\hline Siracusa & 103.501 & 70,6 & 144389 & 66,1 & 138.493 & 62,8 & 3,3 & & \\
\hline
\end{tabular}


TAв. 23 - Elezioni comunali 2008. Sindaci dei comuni capoluogo eletti al primo turno (6 casi).

\begin{tabular}{|c|c|c|c|}
\hline Comune & Sindaco eletto & $\%$ & Sostegno elettorale \\
\hline Treviso & Gobbo & 50,4 & Lista Gentilini; PDL; Lega Nord \\
\hline Brescia & Paroli & 51,4 & $\begin{array}{l}\text { PDL; Lega Nord; UDC; Basta tasse; Partito pensionati; Con lo sport e il } \\
\text { volontariato; PSDI }\end{array}$ \\
\hline Pescara & D'Alfonso & 50,3 & PD; IDV; Pescara città ponte; PS; Insieme per Pescara \\
\hline Catania & Stancanelli & 54,6 & $\begin{array}{l}\text { PDL; MPA-Alleati per il Sud; Lombardo presidente - Sicilia forte e libera; } \\
\text { UDC; Lista per Stancanelli sindaco; Centro democratico siciliano Lista } \\
\text { forzese; Democratici Autonomisti }\end{array}$ \\
\hline Messina & Buzzanca & 50,9 & $\begin{array}{l}\text { MPA - Alleati per il Sud; Conservare il futuro con Briguglio; La Destra- } \\
\text { Gioventù della Libertà; UDC; Forza Azzurri; PRI ; Gli autonomisti } \\
\text { dell'MPA; Dicearco per l'autonomia; il Centro con d'Alia; Rialzati } \\
\text { Messina; PDL }\end{array}$ \\
\hline Siracusa & Visentin & 54,6 & $\begin{array}{l}\text { PDL; UDC; Alleanza Azzurra Sicilia; MPA - Alleati per il Sud; No! } \\
\text { Acquasalata F.I.P.U Movimento Pensionati }\end{array}$ \\
\hline
\end{tabular}

Nota: i nomi in corsivo si riferiscono a presidenti uscenti.

TАВ 24 - Elezioni comunali 2008. Sindaci dei comuni capoluogo eletti al ballottaggio (7 casi).

\begin{tabular}{|c|c|c|c|c|c|c|c|c|}
\hline Comune & $\begin{array}{l}\text { Sindaco } \\
\text { eletto }\end{array}$ & $\begin{array}{c}\% \\
\left(I I^{\circ} \text { t. }\right)\end{array}$ & $\begin{array}{c}\% \\
\text { (I t. })\end{array}$ & Sostegno elettorale & $\begin{array}{l}\text { Candidato } \\
\text { sconfitto }\end{array}$ & $\begin{array}{c}\% \\
\left(I I^{\circ} \text { t. }\right)\end{array}$ & $\begin{array}{c}\% \\
\left(I^{\circ} \text { t. }\right)\end{array}$ & Sostegno elettorale \\
\hline Vicenza & Variati & 50,5 & 31,3 & $\begin{array}{l}\text { PD; Variati sindaco; Lista } \\
\text { Giuliari }\end{array}$ & Sartori & 49,5 & 39,3 & $\begin{array}{l}\text { PDL; Lega Nord; } \\
\text { VicenzaViva }\end{array}$ \\
\hline Sondrio & Molteni & 54,2 & 49,0 & $\begin{array}{l}\text { Sondrio democratica; } \\
\text { PD; Sinistra Arcobaleno; } \\
\text { Sondrio 2020; Sondrio } \\
\text { accesa; PS }\end{array}$ & Faggi & 45,8 & 32,5 & $\begin{array}{l}\text { PDL; Lega Nord; } \\
\text { Faggi sindaco }\end{array}$ \\
\hline Udine & Honsell & 52,8 & 44,2 & $\begin{array}{l}\text { PD; Innovare con } \\
\text { Honsell; Sinistra } \\
\text { Arcobaleno; IDV; } \\
\text { Cittadini per il sindaco }\end{array}$ & Cainero & 47,2 & 39,6 & $\begin{array}{l}\text { Lista Cainero; PDL; } \\
\text { Loego Nord; UDC; } \\
\text { Udine Cainero } \\
\text { Sindaco }\end{array}$ \\
\hline Pisa & Filippeschi & 53,1 & 47,4 & $\begin{array}{l}\text { PD; In lista per Pisa; PS; } \\
\text { IDV }\end{array}$ & $\begin{array}{l}\text { Paoletti } \\
\text { Tangheroni }\end{array}$ & 46,9 & 32,4 & $\begin{array}{l}\text { PDL; Lega Nord; } \\
\text { Rinascita pisana; } \\
\text { Monarchici uniti }\end{array}$ \\
\hline Massa & Pucci & 54,3 & 27,9 & $\begin{array}{l}\text { Pucci sindaco per Massa; } \\
\text { Sinistra Arcobaleno; } \\
\text { Massa al centro; Impegno } \\
\text { per Massa }\end{array}$ & Neri & 45,7 & 38,8 & $\begin{array}{l}\text { PD; PS Socialisti- } \\
\text { Laici-Repubblicani; } \\
\text { IDV; Fare per Massa }\end{array}$ \\
\hline Roma & Alemanno & 53,7 & 40,7 & $\begin{array}{l}\text { PDL; Sindaco Alemanno; } \\
\text { Popolo della vita; MPA; } \\
\text { Voce dei consumatori; } \\
\text { PRI }\end{array}$ & Rutelli & 46,3 & 45,8 & $\begin{array}{l}\text { Per Rutelli; Lista } \\
\text { Bonino-Radicali; } \\
\text { Sinistra Arcobaleno; } \\
\text { IDV; PD; Unione } \\
\text { Democratica per i } \\
\text { consumatori; I } \\
\text { moderati per Roma; } \\
\text { Under } 30\end{array}$ \\
\hline Viterbo & Marini & 62,0 & 49,1 & $\begin{array}{l}\text { PDL; Viterbo vola; Ego } \\
\text { sum Leo }\end{array}$ & Sposetti & 38,0 & 33,5 & PD; Sposetti sindaco \\
\hline
\end{tabular}

Nota: i nomi in corsivo si riferiscono a presidenti uscenti; le liste in corsivo si riferiscono al sostegno elettorale al candidato sopraggiunto al secondo turno. 